\title{
Killing Me Softly: Technology and Privacy
}

\author{
Federica Fornaciari, Ph. D. \\ Assistant Professor, National University, La Jolla, CA
}

\begin{abstract}
What are the frames that emerged in media discourse of privacy during peaks of technological evolution of the 20th and of the 21 st centuries? To address this research question, the current study implements a content analysis of 495 editorials published in major US media outlets during selected decades beginning in the early 20 th century and through 2017.

Relying upon frames identified in a pilot study, the coding protocol aimed at identifying the implementation of the following eight frames: White Lies, Disclosure for Community, Privacy as Property, Appropriate Information Flow, Users' Responsibility, Value of Truth, Privacy is Dead, and Fundamental Privacy. Two coders analyzed the sample reaching high intercoder reliability that ranged between .92 and 1 .

To analyze the data, the author implemented descriptive statistics, Pearson correlation coefficients, and ANOVAs. Findings revealed important longitudinal tendencies. First, the implementation of the frames White Lies and Fundamental Privacy significantly decreased longitudinally whereas Privacy as Property became more present in more recent decades. Second, significant relationships revealed that the frames Appropriate Information Flow, and Disclosure for Community surfaced slightly more frequently in more recent decades. Third, the frame Privacy is Dead surfaced rather steadily as only non-significant differences emerged across timeframes. This media practice suggests a fascinating, dystopian pattern of continuity in the rhetoric used to discuss how newer technologies challenge the flow of personal data, to shed light on the power of new tools over information, and to tackle their role in the claimed death of privacy.
\end{abstract}

Keywords: Frame Theory, Content Analysis, Privacy, Technology 